\title{
BALANCED METRICS ON HARTOGS DOMAINS
}

\author{
ANDREA LOI, MICHELA ZEDDA
}

\begin{abstract}
An $n$-dimensional strictly pseudoconvex Hartogs domain $D_{F}$ can be equipped with a natural Kähler metric $g_{F}$. In this paper we prove that if $m_{0} g_{F}$ is balanced for a given positive integer $m_{0}$ then $m_{0}>n$ and $\left(D_{F}, g_{F}\right)$ is holomorphically isometric to an open subset of the $n$-dimensional complex hyperbolic space.
\end{abstract}

\section{INTRODUCTION}

Let $M$ be a complex manifold endowed with a Kähler metric $g$ and let $\omega$ be the Kähler form associated to $g$, i.e. $\omega(\cdot, \cdot)=g(\cdot, J \cdot)$. Assume that the metric $g$ can be described by a strictly plurisubharmonic real valued function $\Phi: M \rightarrow \mathbb{R}$, called a Kähler potential for $g$, i.e. $\omega=\frac{i}{2} \partial \bar{\partial} \Phi$.

A Kähler potential is not unique, in fact it is defined up to an addition with the real part of a holomorphic function on $M$. Let $\mathcal{H}_{\Phi}$ be the weighted Hilbert space of square integrable holomorphic functions on $(M, g)$, with weight $e^{-\Phi}$, namely

$$
\mathcal{H}_{\Phi}=\left\{\left.f \in \operatorname{Hol}(M)\left|\int_{M} e^{-\Phi}\right| f\right|^{2} \frac{\omega^{n}}{n !}<\infty\right\},
$$

where $\frac{\omega^{n}}{n !}=\operatorname{det}(\partial \bar{\partial} \Phi) \frac{\omega_{0}^{n}}{n !}$ is the volume form associated to $\omega$ and $\omega_{0}=$ $\frac{i}{2} \sum_{\alpha=0}^{n-1} d z_{\alpha} \wedge d \bar{z}_{\alpha}$ is the standard Kähler form on $\mathbb{C}^{n}$. If $\mathcal{H}_{\Phi} \neq\{0\}$ we can pick an orthonormal basis $\left\{f_{j}\right\}$ and define its reproducing kernel by

$$
K_{\Phi}(z, z)=\sum_{j=0}^{\infty}\left|f_{j}(z)\right|^{2} .
$$

Consider the function

$$
\varepsilon_{g}(z)=e^{-\Phi(z)} K_{\Phi}(z, z) .
$$

As suggested by the notation $\varepsilon_{g}$ depends only on the metric $g$ and not on the choice of the Kähler potential $\Phi$. In fact, if $\Phi^{\prime}=\Phi-\operatorname{Re}(\varphi)$, for some holomorphic function $\varphi$, is another potential for $\omega$, we have $e^{-\Phi^{\prime}}=e^{-\Phi}\left|e^{\varphi}\right|^{2}$.

2000 Mathematics Subject Classification. 53C55; 58C25.

Key words and phrases. Kähler metrics; balanced metrics; Hartogs domains.

The first author was supported by the M.I.U.R. Project "Geometric Properties of Real and Complex Manifolds"; the second author was supported by RAS through a grant financed with the "Sardinia PO FSE 2007-2013" funds and provided according to the L.R. $7 / 2007$. 
Furthermore, since $\varphi$ is holomorphic and $\partial \bar{\partial} \Phi^{\prime}=\partial \bar{\partial} \Phi, e^{\varphi}$ is an isomorphism between $\mathcal{H}_{\Phi}$ and $\mathcal{H}_{\Phi^{\prime}}$, and thus we can write $K_{\Phi^{\prime}}(z, z)=\left|e^{\varphi}\right|^{2} K_{\Phi}(z, z)$, where $K_{\Phi}(z, z)$ (resp. $K_{\Phi^{\prime}}(z, z)$ ) is the reproducing kernel of $\mathcal{H}_{\Phi}$ (resp. $\left.\mathcal{H}_{\Phi^{\prime}}\right)$. It follows that $e^{-\Phi(z)} K_{\Phi}(z, z)=e^{-\Phi^{\prime}(z)} K_{\Phi^{\prime}}(z, z)$, as claimed.

In the literature the function $\varepsilon_{g}$ was first introduced under the name of $\eta$-function by J. Rawnsley in [17], later renamed as $\varepsilon$-function in [3], and it is also appear under the name of distortion function for the study of Abelian varieties by J. R. Kempf [14] and S. Ji [13], and for complex projective varieties by S. Zhang [18]. It also plays a fundamental role in the geometric quantization of a Kähler manifold and in the Tian-Yau-Zelditch asymptotic expansion (see [11] and references therein).

Definition. Let $g$ be a Kähler metric on a complex manifold $M$ such that $\omega=\frac{i}{2} \partial \bar{\partial} \Phi$. The metric $g$ is balanced if the function $\varepsilon_{g}$ is a positive constant.

Remark 1. The definition of balanced metrics was originally given by S. Donaldson [7] in the case of a compact polarized Kähler manifold $(M, g)$ and generalized in [2] (see also [4, [10], 9]). In the compact case the potential $\Phi$ will certainly not exist globally and the only holomorphic functions on $M$ are the constants. Nevertheless, since $g$ is polarized there exists an hermitian line bundle $(L, h) \rightarrow M$ such that $\operatorname{Ric}(h)=\omega$. One can then endowed the space of global holomorphic sections of $L$, denoted by $H^{0}(L)$, with the scalar product

$$
\langle s, t\rangle_{h}=\int_{M} h(s(x), t(x)) \frac{\omega^{n}}{n !}, s, t \in H^{0}(L) .
$$

If $H^{0}(L) \neq\{0\}$ one can set

$$
\varepsilon_{g}(x)=\sum_{j=0}^{N} h\left(s_{j}(x), s_{j}(x)\right),
$$

where $\left\{s_{0}, \ldots, s_{N}\right\}, N+1=\operatorname{dim} H^{0}(L)$, is an orthonormal basis of $\left(H^{0}(L),\langle,\rangle_{h}\right)$ and define the metric $g$ balanced if $\varepsilon_{g}$ is a positive constant.

In this paper we study the balanced condition for a particular class of strictly pseudoconvex domains $D_{F}$ of $\mathbb{C}^{n}$, called Hartogs domains (see next section or [8]), equipped with a Kähler metric $g_{F}$ depending on a real valued function $F$. Our main result is Theorem 7 below where we prove that if the metric $m_{0} g_{F}$ of a $n$-dimensional Hartogs domain $D_{F}$ is balanced for a given $m_{0}>n$, then $\left(D_{F}, g_{F}\right)$ is holomorphically isometric to an open subset of the $n$-dimensional complex hyperbolic space. The paper contains another section with the description of the Hartogs domains and the proof of Theorem 7 . 


\section{Statement And PRoof of the main Result}

Let $x_{0} \in \mathbb{R}^{+} \cup\{+\infty\}$ and let $F:\left[0, x_{0}\right) \rightarrow(0,+\infty)$ be a decreasing continuous function, smooth on $\left(0, x_{0}\right)$. The Hartogs domain $D_{F} \subset \mathbb{C}^{n}$ associated to the function $F$ is defined by

$$
D_{F}=\left\{\left.\left(z_{0}, z_{1}, \ldots, z_{n-1}\right) \in \mathbb{C}^{n}|| z_{0}\right|^{2}<x_{0},\|z\|^{2}<F\left(\left|z_{0}\right|^{2}\right)\right\},
$$

where $\|z\|^{2}=\left|z_{1}\right|^{2}+\cdots+\left|z_{n-1}\right|^{2}$. We shall assume that the natural $(1,1)$ form on $D_{F}$ given by

$$
\omega_{F}=\frac{i}{2} \partial \bar{\partial} \log \left(\frac{1}{F\left(\left|z_{0}\right|^{2}\right)-\|z\|^{2}}\right)
$$

is a Kähler form on $D_{F}$. The following proposition gives some conditions on $D_{F}$ equivalent to this assumption:

Proposition 2 ([16]). Let $D_{F}$ be a Hartogs domain in $\mathbb{C}^{n}$. Then the following conditions are equivalent:

(i) the $(1,1)$-form $\omega_{F}$ given by (3) is a Kähler form;

(ii) the function $-\frac{x F^{\prime}(x)}{F(x)}$ is strictly increasing, namely $-\left(\frac{x F^{\prime}(x)}{F(x)}\right)^{\prime}>0$ for every $x \in\left[0, x_{0}\right)$;

(iii) the boundary of $D_{F}$ is strongly pseudoconvex at all $z=\left(z_{0}, z_{1}, \ldots, z_{n-1}\right)$ with $\left|z_{0}\right|^{2}<x_{0}$.

The Kähler metric $g_{F}$ associated to the Kähler form $\omega_{F}$ is the metric we will be dealing with in the present paper. It follows by (3) that a Kähler potential for this metric is given by

$$
\Phi_{F}=-\log \left(F\left(\left|z_{0}\right|^{2}\right)-\|z\|^{2}\right) .
$$

Example 1. When $F(x)=1-x, 0 \leq x<1$,

$$
\left.D_{F}=\mathbb{C H}^{n}=\left\{\left.\left(z_{0}, z_{1}, \ldots, z_{n-1}\right)|| z_{0}\right|^{2}+\|z\|^{2}<1\right)\right\},
$$

the $n$-dimensional complex hyperbolic space $\mathbb{C H}^{n}$ and $g_{F}$ is the hyperbolic metric, i.e. $g_{F}=g_{h y p}$. A Kähler potential for $g_{h y p}$ is given by $\Phi_{h y p}=$ $-\log \left(1-\sum_{\alpha=0}^{n-1}\left|z_{\alpha}\right|^{2}\right)$, and the associated volume form reads

$$
\frac{\omega_{h y p}^{n}}{n !}=\left(1-\sum_{\alpha=0}^{n-1}\left|z_{\alpha}\right|^{2}\right)^{-(n+1)} \frac{\omega_{0}^{n}}{n !} .
$$

Consider $m g_{\text {hyp }}$, for a positive integer $m$, and let $\mathcal{H}_{m \Phi_{\text {hyp }}}$ be the weighted Hilbert space of square integrable holomorphic functions on $\left(\mathbb{C H}^{n}, m g_{\text {hyp }}\right)$, with weight $e^{-m \Phi_{h y p}}=\left(1-\sum_{\alpha=0}^{n-1}\left|z_{\alpha}\right|^{2}\right)^{m}$, namely

$$
\mathcal{H}_{m \Phi_{\text {hyp }}}=\left\{\left.\varphi \in \operatorname{Hol}\left(\mathbb{C H}^{n}\right)\left|\int_{\mathbb{C H}^{n}}\left(1-\sum_{\alpha=0}^{n-1}\left|z_{\alpha}\right|^{2}\right)^{m-(n+1)}\right| \varphi\right|^{2} \frac{\omega_{0}^{n}}{n !}<\infty\right\} .
$$


If $m \leq n$, then it is not hard to see that $\mathcal{H}_{m \Phi_{h y p}}=\{0\}$. On the other hand, for $m>n$, an orthonormal basis for $\mathcal{H}_{m \Phi_{h y p}}$ is given by

$$
\left\{\ldots, \frac{\sqrt{(m+j-1) !}}{\sqrt{\pi^{n}} \sqrt{j_{1} ! \cdots j_{n-1} !(m-n-1) !}} z_{0}^{j_{0}} \cdots z_{n-1}^{j_{n-1}}, \ldots\right\} .
$$

where $j=j_{0}+\cdots+j_{n-1}$. In fact, since the metric depends only on the squared module of the variables, it is easy to see that the monomials $z_{0}^{j_{0}} \cdots z_{n-1}^{j_{n-1}}$ are a complete orthogonal system for $\mathcal{H}_{m \Phi_{\text {hyp }}}$. Further, the following computation

$$
\begin{aligned}
& \int_{\mathbb{C H}^{n}}\left|z_{0}^{j_{0}} \cdots z_{n-1}^{j_{n-1}}\right|^{2}\left(1-\sum_{\alpha=0}^{n-1}\left|z_{\alpha}\right|^{2}\right)^{m-(n+1)} \frac{i^{n}}{2^{n}} d z_{0} \wedge d \bar{z}_{0} \wedge \cdots \wedge d z_{n-1} \wedge d \bar{z}_{n-1} \\
= & \pi^{n} \int_{0}^{1} \cdots \int_{0}^{1-r_{1}-\cdots-r_{n-1}} r_{0}^{j_{0}} \cdots r_{n-1}^{j_{n-1}}\left(1-\sum_{\alpha=0}^{n-1} r_{\alpha}^{j_{\alpha}}\right)^{m-(n+1)} d r_{0} \cdots d r_{n-1} \\
= & \pi^{n} \frac{j_{0} ! \cdots j_{n-1} !(m-n-1) !}{(m+j-1) !}
\end{aligned}
$$

justifies the choice of the normalization constants. The reproducing kernel for $\mathcal{H}_{m \Phi_{\text {hyp }}}$ is then given by

$$
K_{m \Phi_{h y p}}(z, z)=\frac{(m-1) \cdots(m-n)}{\pi^{n}\left(1-\sum_{j=0}^{n-1}\left|z_{j}\right|^{2}\right)^{m}}
$$

and thus

$$
\varepsilon_{m g_{h y p}}(z)=\frac{(m-1) \cdots(m-n)}{\pi^{n}} .
$$

In this example we have that the metric $m g_{h y p}$ is balanced iff $m>n$. In the geometric quantization framework introduced in [3] the Kähler forms satisfying this property play a fundamental role for the quantization by deformation of the Kähler manifold $(M, g)$. In our setting one says that a Kähler manifold $(M, g)$ admits a regular quantization if the functions

$$
\varepsilon_{m g}(z)=e^{-m \Phi(z)} K_{m \Phi}(z, z)
$$

are positive constants (depending on $m$ ) for all sufficiently large positive integers.

Regarding regular quantizations we have the following lemma which will be an important ingredient in the proof of our main result, Theorem 7 ,

Lemma 3. Let $g$ be a Kähler metric on a complex manifold $M$. If $(M, g)$ admits a regular quantization then the scalar curvature of the metric $g$ is constant.

Proof. See Theorem 5.3 in [1] for the compact case and Theorem 4.1 in [15] for the noncompact one. 
Hartogs domains have been considered in [8] and [15] in the framework of quantization of Kähler manifolds. In [5] is studied the existence of global symplectic coordinates on $\left(D_{F}, \omega_{F}\right)$ and [6] deals with the Riemannian geometry of $\left(D_{F}, g_{F}\right)$. In [15] (see also [16]) these domains are studied from the scalar curvature viewpoint. The main results obtained in [16] and in [6] are summarized in the following two lemmata needed in the proof of Theorem 7 and its Corollary 8 .

Lemma 4. Let $\left(D_{F}, g_{F}\right)$ be a n-dimensional Hartogs domain. Assume that its scalar curvatures is constant. Then $\left(D_{F}, g_{F}\right)$ is holomorphically isometric to an open subset of the complex hyperbolic space $\left(\mathbb{C H}^{n}, g_{\text {hyp }}\right)$.

Lemma 5. A Hartogs domain $\left(D_{F}, g_{F}\right)$ is geodesically complete if and only if

$$
\left.\int_{0}^{\sqrt{x_{0}}} \sqrt{-\left(\frac{x F^{\prime}}{F}\right)^{\prime}}\right|_{x=u^{2}} d u=+\infty,
$$

where we define $\sqrt{x_{0}}=+\infty$ for $x_{0}=+\infty$.

For the proof of Theorem 7 we need another result, Lemma 6 below, which is a straightforward generalization to dimension $n$ of Propositions 3.12 and 3.14 proven by M. Engliš in [8]. In order to state it we set

$$
c_{k}\left(F^{m}\right)=\int_{0}^{x_{0}} t^{k} F(t)^{m} G(t) d t
$$

where

$$
G(t)=-\left(\frac{t F^{\prime}}{F}\right)^{\prime}
$$

(notice that $G(t)>0$ by $(i i)$ in Proposition 2) and assume that there exists a real number $\gamma$ such that for all positive integers $m$

$$
\sum_{k=0}^{\infty} \frac{t^{k}}{c_{k}\left(F^{m}\right)}=(m-1+\gamma) F(t)^{-m} .
$$

Many examples of Hartogs domains satisfy this condition (see [8, pp. 450454]). Such domains admit a quantization by deformation (see [8] for details) and so they are also interesting from the physical point of view.

Let us also write the volume element corresponding to the metric $\omega_{F}$ by

$$
\frac{\omega_{F}^{n}}{n !}=\frac{F^{2}\left(\left|z_{0}\right|^{2}\right)}{\left(F\left(\left|z_{0}\right|^{2}\right)-\|\left. z\right|^{2}\right)^{n+1}} G\left(\left|z_{0}\right|^{2}\right) \frac{\omega_{0}^{n}}{n !} .
$$

Lemma 6. Let $\left(D_{F}, g_{F}\right)$ be an Hartogs domain and let $\mathcal{H}_{m \Phi_{F}}$ be the corresponding weighted Hilbert space given by (11). Assume that condition (7) is satisfied for all positive integers $m$. Then $\mathcal{H}_{m \Phi_{F}} \neq\{0\}$ iff $m>n$ and its reproducing kernel is given by

$$
K_{m \Phi_{F}}(z, z)=\frac{(m-2) \cdots(m-n)}{\pi^{n}\left(F\left(\left|z_{0}\right|^{2}\right)-\|z\|^{2}\right)^{m}}[m-1+(1-w) \gamma]
$$


where $w=\frac{\|z\|^{2}}{F\left(\left|z_{0}\right|^{2}\right)}$ and $\gamma$ is the real number appearing in (7).

Proof. It is not hard to verify that the monomials $z_{0}^{j_{0}} z_{1}^{j_{1}} \cdots z_{n-1}^{j_{n-1}}$ are a complete orthogonal system for $\mathcal{H}_{m \Phi_{F}}$, for $m>n$. Hence, the well-known formula for reproducing kernels gives for the Hilbert space $\mathcal{H}_{m \Phi_{F}}$

$$
K_{m \Phi_{F}}(z, z)=\sum_{j_{0}, \ldots, j_{n-1}} \frac{\left|z_{0}\right|^{2 j_{0}} \cdots\left|z_{n-1}\right|^{2 j_{n-1}}}{\left\|z_{0}^{j_{0}} \cdots z_{n-1}^{j_{n-1}}\right\|_{m}^{2}}
$$

where

$$
\left\|z_{0}^{j_{0}} \cdots z_{n-1}^{j_{n-1}}\right\|_{m}^{2}=\int_{D_{F}}\left(F\left(\left|z_{0}\right|^{2}\right)-\|z\|^{2}\right)^{m} \prod_{k=0}^{n-1}\left|z_{k}\right|^{2 j_{k}} \frac{\omega_{F}^{n}}{n !} .
$$

By formula (8) the right hand side is equal to

$$
\int_{D_{F}}\left(F\left(\left|z_{0}\right|^{2}\right)-|| z||^{2}\right)^{m-n-1} \prod_{k=0}^{n-1}\left|z_{k}\right|^{2 j_{k}} F^{2}\left(\left|z_{0}\right|^{2}\right) G\left(\left|z_{0}\right|^{2}\right) \frac{\omega_{0}^{n}}{n !},
$$

which passing to polar coordinates reads

$\pi^{n} \int_{0}^{x_{0}{ }^{1 / 2}} \int_{0}^{F\left(r_{0}^{2}\right)^{1 / 2}} \cdots \int_{0}^{\left(F\left(r_{0}^{2}\right)-\sum_{i=2}^{n-1} r_{i}^{2}\right)^{1 / 2}}\left(F\left(r_{0}^{2}\right)-r^{2}\right)^{m-n-1} \prod_{k=0}^{n-1} r_{k}^{2 j_{k}} F^{2}\left(r_{0}^{2}\right) G\left(r_{0}^{2}\right) 2^{n} d r d r_{0}$,

where $r^{2}=r_{1}^{2}+\cdots+r_{n-1}^{2}, d r=d r_{1} \cdots d r_{n-1}$. Making now the substitution $r_{i}^{2}=t_{i}$ and using again the short notation $t=t_{1}+\cdots+t_{n-1}$, $d t=d t_{1} \cdots d t_{n-1}$, we get

$$
\pi^{n} \int_{0}^{x_{0}} \int_{0}^{F\left(t_{0}\right)} \cdots \int_{0}^{F\left(t_{0}\right)-\sum_{i=2}^{n-1} t_{i}}\left(F\left(t_{0}\right)-t\right)^{m-n-1} \prod_{k=0}^{n-1} t_{k}^{j_{k}} F^{2}\left(t_{0}\right) G\left(t_{0}\right) d t d t_{0},
$$

which substituting $t_{k}=w_{k} F\left(t_{0}\right)$ for $k=1, \ldots, n-1$, becomes

$$
\pi^{n} \int_{0}^{x_{0}} t_{0}^{j_{0}} F\left(t_{0}\right)^{m+j} G\left(t_{0}\right) d t_{0} \int_{0}^{1} \cdots \int_{0}^{1-\sum_{i=2}^{n-1} w_{i}}(1-w)^{m-n-1} \prod_{k=1}^{n-1} w_{k}^{j_{k}} d w,
$$

where again $w=w_{1}+\cdots+w_{n-1}, d w=d w_{1} \cdots d w_{n-1}$. If $m \leq n$ the last integral diverges, so we can assume $m>n$. Therefore,

$$
\left\|z_{0}^{j_{0}} \cdots z_{n-1}^{j_{n-1}}\right\|_{m}^{2}=\pi^{n} \frac{j_{1} ! \cdots j_{n-1} !(m-n-1) !}{(m+j-2) !} c_{j_{0}}\left(F^{m+j}\right)
$$

where $j=j_{1}+\cdots+j_{n-1}$ and $c_{j_{0}}\left(F^{m+j}\right)$ is defined by (5). Thus

$$
K_{m \Phi_{F}}(z, z)=\sum_{j_{0}, \ldots, j_{n-1}}\left|z_{0}\right|^{2 j_{0}} \cdots\left|z_{n-1}\right|^{2 j_{n-1}} \frac{(m+j-2) !}{\pi^{n} j_{1} ! \cdots j_{n-1} !(m-n-1) !}\left(c_{j_{0}}\left(F^{m+j}\right)\right)^{-1} .
$$


By (7) we can carry out the summation over $j_{0}$, getting

$$
\begin{aligned}
K_{m \Phi_{F}}(z, z) & =\sum_{j_{1} \ldots, j_{n-1}}\left|z_{1}\right|^{2 j_{1}} \cdots\left|z_{n-1}\right|^{2 j_{n-1}} \frac{(m+j-2) !(m+j-1+\gamma)}{\pi^{n} j_{1} ! \cdots j_{n-1} !(m-n-1) !} F^{-m-j}\left(\left|z_{0}\right|^{2}\right) \\
& =\sum_{j_{1} \ldots, j_{n-1}} \frac{\left|z_{1}\right|^{2 j_{1}}}{F^{j_{1}}\left(\left|z_{0}\right|^{2}\right)} \cdots \frac{\left|z_{n-1}\right|^{2 j_{n-1}}}{F^{j_{n-1}}\left(\left|z_{0}\right|^{2}\right)} \frac{(m+j-2) !(m+j-1+\gamma)}{\pi^{n} j_{1} ! \cdots j_{n-1} !(m-n-1) !} F^{-m}\left(\left|z_{0}\right|^{2}\right) \\
& =\sum_{j_{1} \ldots, j_{n-1}} w_{1}^{j_{1}} \cdots w_{n-1}^{j_{n-1}} \frac{(m+j-2) !(m+j-1+\gamma)}{\pi^{n} j_{1} ! \cdots j_{n-1} !(m-n-1) !} F^{-m}\left(\left|z_{0}\right|^{2}\right) \\
& =\frac{(m-2) \cdots(m-n)}{\pi^{n}} \frac{w_{1}^{j_{1}}}{j_{1} !} \cdots \frac{w_{n-1}^{j_{n-1}}}{j_{n-1} !}\left[\left(\begin{array}{c}
m+j-1 \\
m-1
\end{array}\right)(m-1)+\right. \\
& =\frac{(m-2) \cdots(m-n)}{\pi^{n}}\left[\frac{m-1}{(1-w)^{m}}+\frac{\gamma}{(1-w)^{m-1}}\right] F^{-m}\left(\left|z_{0}\right|^{2}\right) \\
& =\frac{(m-2) \cdots(m-n)}{\pi^{n}}\left[\frac{m-1}{\left(F\left(\left|z_{0}\right|^{2}\right)-|| z \|^{2}\right)^{m}}+\frac{(1-w) \gamma}{\left(F\left(\left|z_{0}\right|^{2}\right)-\|z\|^{2}\right)^{m}}\right] \\
& =\frac{(m-2) \cdots(m-n)}{\pi^{n}\left(F\left(\left|z_{0}\right|^{2}\right)-\|z\|^{2}\right)^{m}}[m-1+(1-w) \gamma] .
\end{aligned}
$$

We can now state and prove our main result, which characterizes the hyperbolic space among Hartogs domains in terms of a balanced condition.

Theorem 7. Let $\left(D_{F}, g_{F}\right)$ be a n-dimensional Hartogs domain. Assume that condition (7) is satisfied for all positive integers $m$. If $m_{0} g_{F}$ is balanced then $m_{0}>n$ and $\left(D_{F}, g_{F}\right)$ is holomorphically isometric to an open subset of the complex hyperbolic space $\left(\mathbb{C H}^{n}, g_{\text {hyp }}\right)$.

Proof. Since by Lemma $6 \mathcal{H}_{m \Phi_{F}}=\{0\}$ for $m_{0} \leq n$, we can set $m_{0}>$ $n$. Assume that $m_{0} g_{F}$ is balanced, namely $e^{m_{0} \Phi_{F}}=c_{m_{0}} K_{m_{0} \Phi_{F}}$, for some positive constant $c_{m_{0}}$. Therefore,

$$
\left(F\left(\left|z_{0}\right|^{2}\right)-\|\left. z\right|^{2}\right)^{-m_{0}}=c_{m_{0}} K_{m_{0} \Phi_{F}}(z, z) .
$$

By Lemma 6 we get

$$
\left(F\left(\left|z_{0}\right|^{2}\right)-\|z\|^{2}\right)^{-m_{0}}=c_{m_{0}} \frac{\left(m_{0}-2\right) \cdots\left(m_{0}-n\right)}{\pi^{n}\left(F\left(\left|z_{0}\right|^{2}\right)-\|\left. z\right|^{2}\right)^{m_{0}}}\left[m_{0}-1+(1-w) \gamma\right],
$$

that is

$$
\pi^{n}=c_{m_{0}}\left(m_{0}-2\right) \cdots\left(m_{0}-n\right)\left[m_{0}-1+(1-w) \gamma\right],
$$

which yelds $\gamma=0$, being $(1-w) \gamma$ the only term depending on the variables. Since $\gamma$ is fixed for all $m$, it follows that the reproducing kernel of $\mathcal{H}_{m \Phi_{F}}$, 
for $m>n$, is given by

$$
K_{m \Phi_{F}}(z, z)=\frac{(m-1)(m-2) \cdots(m-n)}{\pi^{n}\left(F\left(\left|z_{0}\right|^{2}\right)-\|z\|^{2}\right)^{m}} .
$$

By (2), we have

$$
\varepsilon_{m g_{F}}(z)=K_{m \Phi_{F}}(z, z)\left(F\left(\left|z_{0}\right|^{2}\right)-\|z\|^{2}\right)^{m}=\frac{(m-1)(m-2) \cdots(m-n)}{\pi^{n}} .
$$

Hence, for all $m>n,\left(D_{F}, g_{F}\right)$ admits a regular quantization. By Lemma 3 and Lemma 4 above, $\left(D_{F}, g_{F}\right)$ is then holomorphically isometric to an open subset of the complex hyperbolic space.

Combining Lemma 5 with Theorem 7 one gets:

Corollary 8. Let $\left(D_{F}, g_{F}\right)$ be an n-dimensional Hartogs domain. Assume that conditions (4) and (7) are satisfied (the latter for all positive integers $m)$. If for some positive integer $m_{0}, m_{0} g_{F}$ is a balanced metric then $\left(D_{F}, g_{F}\right)$ is holomorphically isometric to the complex hyperbolic space $\left(\mathbb{C H}^{n}, g_{\text {hyp }}\right)$.

Remark 9. A balanced metric $g$ on a complex manifold $M$ is projectively induced. Indeed, there exists a holomorphic map $f: M \rightarrow \mathbb{C P}^{\infty}$, called the coherent states map in J. Rawnsley terminology [17, into the infinite dimensional complex projective space $\mathbb{C P}^{\infty}$ such that $f^{*} g_{F S}=g$, where $g_{F S}$ denotes the Fubini-Study metric on $\mathbb{C P}^{\infty}$ (see [2] for details). Not all projectively induced metrics are balanced. Indeed, there exist $n$-dimensional Hartogs domains $\left(D_{F}, g_{F}\right), D_{F} \neq \mathbb{C H}^{n}$, where $m_{0} g_{F}$ is projectively induced for $m_{0}>n$. An example is given by the so called Springer domain $\left(D_{F}, g_{F}\right)$ corresponding to the function $F(x)=e^{-x}, x \in[0,+\infty)$ (see [12])). Moreover, it is not hard to verify that this domain satisfies condition (17) in Theorem 7 with $\gamma=1$ (see also [8]). This shows that the condition that $m_{0} g_{F}$ is balanced in Theorem 7 cannot be replaced by the weaker condition that $m_{0} g_{F}$ is projectively induced.

\section{REFERENCES}

[1] C. Arezzo, A. Loi, Quantization of Kähler manifolds and the asymptotic expansion of Tian-Yau-Zelditch, J. Geom. Phys. 867 (2003), 1-13.

[2] C. Arezzo, A. Loi, Moment maps, scalar curvature and quantization of Kähler manifolds, Comm. Math. Phys. 243 (2004), 543-559.

[3] M. Cahen, S. Gutt, J. Rawnsley, Quantization of Kähler manifolds. I: Geometric interpretation of Berezin's quantization, J. Geom. Physics 7 (1990), 45-62.

[4] F. Cuccu and A. Loi, Global symplectic coordinates on complex domains, J. Geom. and Phys. 56 (2006), 247-259.

[5] F. Cuccu and A. Loi, Balanced metrics on $\mathbb{C}^{n}$, J. Geom. Phys. 57 (2007), 1115-1123.

[6] A. J. Di Scala, A. Loi and F. Zuddas, Riemannian geometry of Hartogs domains, Int. J. Math. 2, vol. 20 (2009), 139-148. 
[7] S. Donaldson, Scalar Curvature and Projective Embeddings, I, J. Diff. Geometry 59 (2001), 479-522.

[8] M. Engliš, Berezin quantization and reproducing kernels on complex domains, Trans. of the AMS no. 2, vol. 348 (1996), 411-479.

[9] M. Engliš, Weighted Bergman kernels and balanced metrics, RIMS Kokyuroku 1487 (2006), 40-54.

[10] A. Greco, A. Loi, Radial balanced metrics on the unit disk J. Geom. Phys. 60 (2010), 53-59.

[11] T. Gramchev, A. Loi, TYZ expansion for the Kepler manifold, Comm. Math. Phys. 289, (2009), 825-840.

[12] A. Loi, Holomorphic maps of Hartogs domains into complex space forms, Riv. Mat. Univ. Parma (7) vol. 1 (2002), 103-113.

[13] S. Ji, Inequality for distortion function of invertible shaves on Abelian varieties, Duke Math. J. 58 (1989), 657-667.

[14] G. R. Kempf, Metrics on invertible shaves on abelian varieties, Topics in algebraic geometry (Guanajuato, 1989), Aportationes Mat. Notas Investigacion 5, Soc. MAt. Mexicana, Mexico, 1992, pp. 107-108.

[15] A. Loi, Regular quantizations of Kähler manifolds and constant scalar curvature metrics, J. of Geom. Phys. 53 (2005), 354-364.

[16] A. Loi, F. Zuddas, Canonical metrics on Hartogs domains, to appear in Osaka Journal of Mathematics.

[17] J. Rawnsley, Coherent states and Kähler manifolds, Quart. J. Math. Oxford (2), n. 28 (1977), 403-415.

[18] S. Zhang, Heights and reductions of semi-stable varieties, Comp. Math. 104 (1996), 77-105.

Dipartimento di Matematica e Informatica, Università di Cagliari, Via OsPEDALE 72, 09124 CAGLiari, ItAly

E-mail address: loi@unica.it; michela.zedda@gmail.com 